\title{
Genetic changes in the EPAS1 gene between Tibetan and Han ethnic groups and adaptation to the plateau hypoxic environment
}

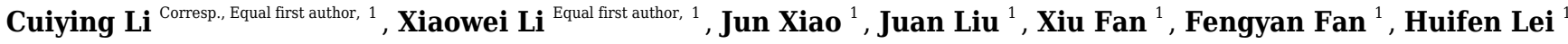 \\ ${ }^{1}$ Department of Blood Transfusion, Air Force Medical Center, PLA, Beijing, China \\ Corresponding Author: Cuiying Li \\ Email address: licuiying2017@126.com
}

In the Chinese Han population, prolonged exposure to hypoxic conditions can promote compensatory erythropoiesis which improves hypoxemia. However, Tibetans have developed unique phenotypes, such as downregulation of the hypoxia-inducible factor pathway through EPAS1 gene mutation, thus the mechanism of adaption of the Han population should be further studied. The results indicated that, under plateau hypoxic conditions, the plains population was able to acclimate rapidly to hypoxia through increasing EPAS1 mRNA expression and changing the hemoglobin conformation. Furthermore, the mutant genotype frequencies of the rs13419896, rs1868092 and rs4953354 loci in the EPAS1 gene were significantly higher in the Tibetan population than in the plains population. The EPAS1 gene expression level was lowest in the Han population carrying the A-A homozygous mutant of the rs13419896 locus but that it increased rapidly after these individuals entered the plateau. At this time, the hemoglobin content was lower in the homozygous mutant Han group than in the wild-type and heterozygous mutant populations, and the viscosity of blood was reduced in populations carrying the A-A haplotypes in rs13419896 and rs1868092. Among Tibetans, the group carrying homozygous mutations of the three SNPs also had lower hemoglobin concentrations than the wild-type. The Raman spectroscopy results showed that exposure of the Tibetan and Han population to hypoxic conditions changed the spatial conformation of hemoglobin and its binding ability to oxygen. The Tibetan population has mainly adapted to the plateau through genetic mutations, whereas some individuals adapt through changes in hemoglobin structure and function. 


\section{Genetic changes in the EPAS1 gene between Tibetan and}

\section{Han ethnic groups and adaptation to the plateau hypoxic}

\section{3 environment}

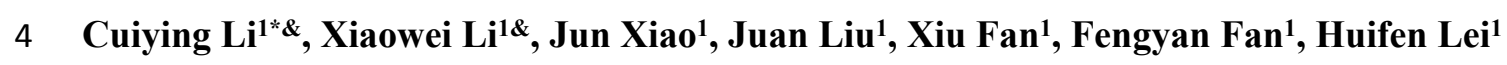

5 1Department of Blood Transfusion, Air Force Medical Center, PLA, 30 Fucheng Road, Beijing

6 100142, P R China

$7 \quad$ \& Cuiying Li and Xiaowei Li contributed equally to this study

8 * Correspondence:

9 Cuiying $\mathrm{Li}$

30 Fucheng Road, Beijing 100142, P R China

11 Email address: licuiying2017@126.com

\section{Abstract}

In the Chinese Han population, prolonged exposure to hypoxic conditions can promote compensatory erythropoiesis which improves hypoxemia. However, Tibetans have developed unique phenotypes, such as downregulation of the hypoxia-inducible factor pathway through EPAS1 gene mutation, thus the mechanism of adaption of the Han population should be further studied. The results indicated that, under plateau hypoxic conditions, the plains population was able to acclimate rapidly to hypoxia through increasing EPAS1 mRNA expression and changing the hemoglobin conformation. Furthermore, the mutant genotype frequencies of the rs13419896, rs 1868092 and rs4953354 loci in the EPAS1 gene were significantly higher in the Tibetan population than in the plains population. The EPAS1 gene expression level was lowest in the Han population carrying the A-A homozygous mutant of the rs13419896 locus but that it increased rapidly after these individuals entered the plateau. At this time, the hemoglobin content was lower in the homozygous mutant Han group than in the wild-type and heterozygous mutant populations, 
and the viscosity of blood was reduced in populations carrying the A-A haplotypes in rs13419896 and rs1868092. Among Tibetans, the group carrying homozygous mutations of the three SNPs also had lower hemoglobin concentrations than the wild-type. The Raman spectroscopy results showed that exposure of the Tibetan and Han population to hypoxic conditions changed the spatial conformation of hemoglobin and its binding ability to oxygen. The Tibetan population has mainly adapted to the plateau through genetic mutations, whereas some individuals adapt through changes in hemoglobin structure and function.

\section{Introduction}

The Qinghai-Tibet Plateau is located in the western part of China and has an average elevation of 4,000 meters. It is characterized by low pressure, low oxygen, low temperature, high winds, dry climate, strong ultraviolet radiation and other unfavorable factors. The oxygen content is only $60 \%$ of that of the plains, and millions of annual trips are made to the Qinghai-Tibet Plateau annually, presenting a serious environmental challenge for humanity(Petousi et al. 2014). In addition, some people must live in the plateau environment for a long time. Prolonged exposure to hypoxic conditions can promote compensatory erythropoiesis, increased hemoglobin levels, and an increased blood oxygen capacity, all of which improve hypoxemia. Upon initially entering the plateau, members of the plains population may experience acute altitude sickness due to a lack of oxygen, with main manifestations of palpitation, shortness of breath, headaches, and sleep disturbances. In severe, high-altitude brain edema or pulmonary edema and death may occur (Meier et al. 2017). During a prolonged stay in the plateau, the above-mentioned symptoms of adverse reactions may ease or disappear. If the above symptoms persist for more than three months, red blood cells will continue to proliferate, and the condition can develop into chronic high plateau diseases such as high plateau polycythemia. However, Tibetan people who have lived in the plateau for generations have a unique set of plateau-adapted physiological characteristics: their arterial oxygen content is basically unchanged, resting ventilation is increased, birth weight is increased, and hemoglobin concentration is low(Bigham \& Lee 2014). Recent studies have shown that these physiological changes are closely related to the genetic characteristics of the population, 
53 highlighting the importance of genetic adaptation and not solely phenotypic changes in plateau 54 adaptation(Bigham \& Lee 2014).

55 Genomics, transcriptomic microarray, and whole-genome linkage analysis have revealed that 56 hypoxia inducible factor (HIF)-1 and HIF-2 plays an important role in altitude hypoxia adaptation(Lendahl et al. 2009). Studies have shown that HIF is widely expressed in mammals and that it plays a key role in hypoxia and can promote the adaptation of mammals to high altitude environments (Bigham \& Lee 2014). The most important study of HIF focused on endothelial PAS domain protein 1 (EPAS1), which encodes HIF-2 $\alpha$; several single nucleotide polymorphisms (SNPs) in EPAS1 is closely related to the low level of hemoglobin ( $\mathrm{Hb})$ in Tibetan populations (Beall et al. 2010; Xu et al. 2015). Specifically, rs13419896, rs1868092, and rs4953354 are great difference between Tibetan and Han populations (Peng et al. 2011), and are also significantly correlated with low hemoglobin (Beall et al. 2002; Chen et al. 2014). EPAS1 distribution is tissuespecific; it is mainly expressed in tissues and organs related to metabolism and the oxygen supply, such as placenta,vascular endothelium (Hu et al. 2003) and kidney(Lee \& Percy 2011). Therefore, it was inferred that EPAS1 expression plays an important role in oxygen metabolism and sensing. The most striking differences between Han people who acutely enter the plateau and Tibetans whose families have lived in the plateau for generations are related to $\mathrm{Hb}$. Why do some people experience sickness after acutely entering the plateau? Can the Han population show acclimation after living in the plateau for a long time? To answer these questions, we tested the physiological indicators of three groups of people. We found that there was no significant difference in $\mathrm{Hb}$ concentration between the Tibetans and plains population, whereas the number of red blood cells and the $\mathrm{Hb}$ level of people who acutely enter the plateau and those who lived in the plateau for a long time were significantly higher than those of the plains. The blood oxygen saturation level $\left(\mathrm{SpO}_{2}\right)$ of people acutely entering the plateau and those who had lived in the plateau for a long time were significantly lower than that of the plains population, while the level of Tibetans was slightly lower than that of the plains population. Compared with the plains population, the affinity of $\mathrm{Hb}$ for oxygen $\left(\mathrm{P}_{50}\right)$ increased rapidly in people who acutely entered the plateau and was lower 
80 in the Tibetan population and in those who had lived in the plateau for a long time(Li et al. 2018).

81 To clarify the mechanism and identify detection and prevention measures for the plains population

82 prior to entry into the plateau, we conducted a series of studies at the molecular level focusing on

83 the genetic and epigenetic differences in the EPASl gene and on $\mathrm{Hb}$ conformations.

\section{Materials and Methods}

85

86

\section{Materials}

(1) We selected 155 Tibetans whose families had lived at high altitude (3700 meters above sea level, Lhasa City, Tibet, China) for generations. This group of individuals included 155 males aged 18-57 years, with an average age of $(33.9 \pm 8.3)$; (2) we also selected 190 Han male people whose families had lived in the plains (50-500 meters above sea level) for generations; this population aged 13-55 years, (average age 34.7 \pm 9.2 years); Four additional population were included in the study:(3) 50 plains Han people who remained in the plateau at 4300 meters above sea level for three days; (4) 50 plains Han people who remained in the plateau at 4300 meters above sea level for seven days; (5) 44 plains Han people who remain in the plateau at 3700 meters above sea level for 30 days; and (6) 24 Han men who had lived in the plateau for a long time (>10 years, 3700 meters above sea level). Group (3), (4), and (5) consisted of the same group of men (Table 1). This study was approved by the Institutional Review Board of Air Force Medical Center, PLA (2017-05-YJ01) and the research was carried out in accordance with the World Medical Association Declaration of Helsinki. All volunteers signed informed consent forms, and personal background investigations were conducted to rule out kinship among them.

\section{Monitoring of routine physiological indicators}

Routine blood examination was performed for 155 subjects in group (1), 190 subjects in group (2), 50 subjects in group (3), 50 subjects in group (4), 44 subjects in group (5), and 24 subjects in group (6) using the automatic whole blood cell analyzer (Model TEK3600, Tecom Science Co., Ltd., China). For 50 of the subjects in group (1) through (3), 44 of the subjects in group (5) and 10 of the subjects in group (6), the blood oxygen saturation level was measured with a portable blood oxygen saturation detector. 
107

108

109

110

111

112

113

114

115

116

117

118

119

120

121

122

123

124

125

126

127

128

129

130

131

132

133

\section{Detection of mutation loci in the EPAS1 gene}

DNA was isolated from $200 \mu \mathrm{L}$ of EDTA-anticoagulated peripheral blood collected from each of 100 subjects in group (1), 100 subjects in group (2), and 50 subjects in group (6). Genomic DNA was extracted using a blood genomic DNA extraction kit according to directions provided with the kit (Tiangen Bio Inc., Beijing, China).

Design of the primer sequence corresponding to the EPAS1 gene sequence was based on information obtained from NBCI. The following primers were designed and used in amplification and sequencing based on the information for the rs13419896, rs1868092, and rs4953354: F1: 5'TCATTCCCTGTTCCCTCCTCCTT-3', R1: 5'- GCCAGCTTCCCTTGACCATCTT -3'; F2: 5'TGAGCTGATAAGACTGGTGA-3'， R2： 5'-AAGTACATGCTGCTGGAATG-3'; F3： 5'AGAGGGAATCCAGTGTGAGG-3', R3: 5'-GGGAGTGGTGATGAAAGAAG-3'.

The above mutation sites of the EPAS1 gene were detected by PCR and direct sequencing. The PCR reaction system consisted of $10 \mu \mathrm{L}$ of PCR premix, $0.5 \mu \mathrm{L}$ of each upstream and downstream primers $(10 \mu \mathrm{M}), 2 \mu \mathrm{L}$ of template, and water added to a total volume of $20 \mu \mathrm{L}$. The reaction conditions were as follows: pre-denaturation at $95^{\circ} \mathrm{C}$ for 3 minutes, 28 cycles of denaturation at $95^{\circ} \mathrm{C}$ for 5 seconds, annealing at $57^{\circ} \mathrm{C}$ for 20 seconds, and extension at $72^{\circ} \mathrm{C}$ for 20 seconds. The resulting PCR products were sent directly to SinoGenoMax Co., Ltd, China, for sequencing.

\section{RT-qPCR measeurement of EPAS1 gene expression level in each group}

Total RNA was extracted from $400 \mu \mathrm{l}$ of EDTA-K 2 anticoagulated peripheral blood according to the procedures supplied in the manual of a Whole Blood RNA Extraction Kit (Life Technology). RNA with good purity $\left(\mathrm{OD}_{260} / \mathrm{OD}_{280}\right.$ between 1.7 and 2.0$)$ was used in subsequent experiments.

Reverse transcription was performed using ReverTra Ace ${ }^{\circledR}$ qRCR RT Master Mix with gDNA Remover (Toyobo Co. Ltd.,) in a reaction consisting of $2 \mu \mathrm{L}$ of DNA Mix (with gRNA Remover added), $2 \mu \mathrm{L}$ of RT Master Mix II, $500 \mathrm{ng}$ of RNA template, and water added to a total volume of $10 \mu \mathrm{L}$. The reverse transcription reaction was performed at $37^{\circ} \mathrm{C}$ for 15 minutes, $50^{\circ} \mathrm{C}$ for 5 minutes, and $98^{\circ} \mathrm{C}$ for 5 minutes, and the obtained cDNA was stored at $-20^{\circ} \mathrm{C}$.

PCR primers were designed based on the information available in National Center for 
134

135

136

137

138

139

140

141

142

143

144

145

146

147

Biotechnology Information (NCBI) database, as follows: upstream primer: 5'TTGATAGCAGTGGCAAGGGG-3', downstream primer: 5'-GAGATGATGGCGTCTCCTGG3'; upstream primer of the reference gene RPL13A: 5'-AAAAGCGGATGGTGGTTCC-3', downstream primer of the reference gene RPL13A: 5'-GCTGTCACTGCCTGGTACTT-3'. The reaction system consisted of $10 \mu \mathrm{L}$ of SYBR Green premix, $0.5 \mu \mathrm{L}$ each of the upstream and downstream primers $(10 \mu \mathrm{M}), 2 \mu \mathrm{L}$ of template, and water added to a total of $20 \mu \mathrm{L}$. The PCR program was as follows: pre-denaturation at $95^{\circ} \mathrm{C}$ for 3 minutes and then $95^{\circ} \mathrm{C}$ for 5 seconds, $57^{\circ} \mathrm{C}$ for 20 seconds, and $72^{\circ} \mathrm{C}$ for 20 seconds for 40 cycles. Melting curve analysis and PCR data analysis were performed using the Bio-Rad CFX96 program.

\section{Raman spectroscopy analysis of Hb conformation}

We selected ten individuals in group (1), three in group (2), three in group (3), three in group (4), three in group (5), and three in group (6); all of these male individuals were selected by random number generator. The red blood cells obtained from these subjects were mixed with glycerin, and the samples were loaded into a 96-well plate (approximately $200 \mu \mathrm{L} /$ well). Raman spectrometry was set at an excitation wavelength of $514 \mathrm{~nm}$, power of $2 \mathrm{~mW}$, and an exposure time of $30 \mathrm{~s}$ and was repeated twice. The incident laser was focused on a point $300 \mu \mathrm{m}$ below the liquid surface. The Raman spectra were measured using a 20x lens. The obtained Raman spectral data were subjected to baseline calibration using Origin 8.0 software and smoothed by the Savitzky-Golay method.

\section{Data analysis}

SPSS 20.0 statistical software was used for data processing and statistical analysis. Multiple groups were compared using one-way analysis of variance (ANOVA). Dunnett's test was used for comparisons between groups. $P<0.05$ was considered to indicated statistical significance.

\section{Results}

Differences in physiological indexes between Tibetan and Han ethnic groups in the plateau hypoxic environment 
160

161

162

163

164

165

166

167

168

169

170

171

172

173

174

175

176

177

178

179

180

181

182

183

184

185

186

In order to reveal the mechanism of plateau adaptation difference between Tibetan and Han population, we first compared the $\mathrm{Hb}$ concentration changes among these two groups. Compared with the $\mathrm{Hb}$ level of the Han population in the plains $(127-179 \mathrm{~g} / \mathrm{L}, 152.4 \pm 9.1 \mathrm{~g} / \mathrm{L})$, the Hb level of the Tibetan (120-180 g/L, 148.4 $\pm 15.6 \mathrm{~g} / \mathrm{L})$ did not show significant changes; however, Hb level of Han individuals who acutely entered the plateau for three days (153-197 g/L, 171.8 $\pm 11.0 \mathrm{~g} / \mathrm{L}$ ) was significantly increased. After these individuals had remained in the plateau for a prolonged period, their Hb level (150-201 g/L, 175.9 $\pm 10.3 \mathrm{~g} / \mathrm{L})$ remained relatively high. In Han individuals who had lived in the plateau for a long time (>10 years), the Hb concentration (124-201 g/L, 171.1 $\pm 9.149 \mathrm{~g} / \mathrm{L}$ ) was slightly decreased, but it was still higher than that of the general Han populations (Fig. 1A).

$\mathrm{SpO}_{2}$ was significantly lower in individuals who acutely entered the plateau for three days (79$93 \%, 87.59 \pm 3.10 \%$ ) than in the plains population $(94-99 \%, 97.28 \pm 1.46 \%) . \mathrm{SpO}_{2}$ increased slightly in individuals who had acclimated to the plateau for 30 days $(85-95 \%, 90.36 \pm 2.57 \%)$ but was still lower than that in the plains group. The $\mathrm{SpO}_{2}$ of Han individuals who had lived in the plateau for a long time $(80-95 \%, 87.70 \pm 5.56 \%)$ was similar to that of the group that acutely entered the plateau for three days. Compared with the plains group, the $\mathrm{SpO}_{2}$ of Tibetan (90- 99\%, 94.55 $\pm 2.35 \%$ ) was lower than that of the plains group but significantly higher than that of other groups (not labeled) (Fig. 1 B).

Differences in $E P A S 1$ gene polymorphism, and mRNA expression between Tibetan and Han ethnic groups

As an important transcription factor of HIF-2 $\alpha, E P A S 1$ directly activates the downstream expression of erythropoietin (EPO), which in turn leads to an increase in erythropoiesis. This is the basis for adaptation in people who initially enter the plateau (Simonson et al. 2015). EPASI gene polymorphism and expression are directly related to the $\mathrm{Hb}$ concentration and blood oxygen saturation in Tibetans who are indigenous to the plateau (Peng et al. 2017). To elucidate the mechanism and significance of the above-mentioned changes in physiological indexes under hypoxic conditions in the plateau, we further compared the genetic changes in the EPASI gene 
187

188

189

190

191

192

193

194

195

196

197

198

199

200

201

202

203

204

205

206

207

208

209

210

211

212

between the Tibetan and Han populations to elucidate the theoretical basis for the prevention and treatment of altitude sickness in the plains population.

\section{Distribution of EPAS1 gene polymorphisms in Tibetan and Han populations}

Three different SNPs of the EPAS1 gene were founded in different subpopulations. As shown in Table 2, the frequencies of the A allele of the rs 13419896 locus were $86.1 \%, 32.6 \%$ in the Tibetans and the Han population, respectively. In these population, the frequencies of the "G-A" and " $\mathrm{A}$ A" genotypes were $22.6 \%, 47.4 \%$ and $74.8 \%, 8.9 \%$, respectively; the frequencies of the A allele of the rs1868092 locus were $72.6 \%$ and, respectively, and the frequencies of the "G-A" and " $\mathrm{A}$ A" genotype were $39.4 \%, 19.5 \%$ and $52.9 \%, 0.5 \%$, respectively; the frequencies of the G allele of the rs4953354 locus were $75.5 \%$ and $12.6 \%$, respectively; and the frequencies of the "G-A" and "G-G" genotypes were $25.8 \%, 23.1 \%$ and $62.6 \%, 10.1 \%$, respectively. There were statistically significant differences in the frequencies of the three SNPs between the Tibetan and Han groups. Therefore, the genotype with homozygous mutant alleles in the three SNPs is called the plateauadaptive genotype.

\section{Differences in EPAS1 mRNA expression between the Tibetan and Han populations}

Real-time quantitative PCR was used to measure the EPAS1 gene expression levels in Han subjects whose families had lived in the plains for generations, Han subjects who entered and remained in the plateau for various time periods, Han subjects living in the plateau for a long time, and Tibetan. The results showed no change EPAS1 gene expression occurred in individuals who acutely entered the plateau for three days; however, the EPAS1 mRNA level was significantly increased at seven days, but decreased after 30 days of acclimation in the plateau, and increased again in individuals who had lived in the plateau for a long time( $>10$ years). However, there was no significant difference in EPAS1 mRNA levels between the Tibetan and Han population (Fig. 2).

Effect of genetic alteration of the EPAS1 gene in the plateau hypoxic environment on the physiological indexes of Tibetan and Han peoples

\section{Effect of EPAS1 polymorphism on Hb and blood oxygen saturation levels}


213 Then, in order to investigate different physiological indexes in the Tibetan groups and in Han 214 populations, stratified analysis was used based on the three genotypes in the EPAS1 gene. The 215 results showed that Han individuals of the plains population with various genotypes showed no 216 significant difference in $\mathrm{Hb}$ levels before entering the plateau. However, when the Han groups 217 entered the plateau, the $\mathrm{Hb}$ concentrations of the plateau-adaptive genotype with homozygous 218 alleles in the three SNPs were lower than those of the other two genotypes. In Han groups, the 219 average $\mathrm{Hb}$ concentration of the homozygous mutant of the rs 13419896 site $(170 \mathrm{~g} / \mathrm{L})$ was lower 220 than the wild-type $(173 \mathrm{~g} / \mathrm{L})$ and that of the heterozygous mutant $(171 \mathrm{~g} / \mathrm{L})$; the average $\mathrm{Hb}$ 221 concentration of the homozygous mutant of the rs1868092 site was $164 \mathrm{~g} / \mathrm{L}$, which was 222 significantly lower than that of the wild-type at $172 \mathrm{~g} / \mathrm{L}$ and that of the heterozygous mutant at 172 $223 \mathrm{~g} / \mathrm{L}$; the average $\mathrm{Hb}$ concentration of the homozygous mutant of the rs4953354 site was $169 \mathrm{~g} / \mathrm{L}$, 224 lower than both that of the wild-type at $174 \mathrm{~g} / \mathrm{L}$ and the heterozygous mutant at $174 \mathrm{~g} / \mathrm{L}$. The

225

226

227

228

229

230

231

232

233

234

235

236

237

238

239 average $\mathrm{Hb}$ concentration of the plateau-adaptive genotype of the rs 13419896 site in Han living in the plateau (>10 years) was $158 \mathrm{~g} / \mathrm{L}$, significantly lower than both the wild-type at $174 \mathrm{~g} / \mathrm{L}$ and the heterozygous mutant at $171 \mathrm{~g} / \mathrm{L}$. In Tibetans, the $\mathrm{Hb}$ concentrations of the plateau-adaptive genotype with homozygous alleles in the three SNPs were lower than the wild-type and that of the heterozygous mutant; the average $\mathrm{Hb}$ concentration of the homozygous mutant of the rs13419896 site was $153 \mathrm{~g} / \mathrm{L}$, significantly lower than the wild-type at $172 \mathrm{~g} / \mathrm{L}$ and the heterozygous mutant at $158 \mathrm{~g} / \mathrm{L}$; the average $\mathrm{Hb}$ concentration of the homozygous mutant at the rs 1868092 site was 150 $\mathrm{g} / \mathrm{L}$, significantly lower than the wild-type at $189 \mathrm{~g} / \mathrm{L}$ and the heterozygous mutant at $154 \mathrm{~g} / \mathrm{L}$; the average $\mathrm{Hb}$ concentration of the homozygous mutant of the rs4953354 site was $153 \mathrm{~g} / \mathrm{L}$, significantly lower than the wild-type at $160 \mathrm{~g} / \mathrm{L}$ and the heterozygous mutant at $158 \mathrm{~g} / \mathrm{L}$ (Table 3). These findings indicated that genetic mutations in the plains Han Chinese also have an effect on the $\mathrm{Hb}$ concentration. However, the association of these mutations with blood oxygen saturation did not differ among all the populations (Table 4).

\section{Effect of EPAS1 gene polymorphism on mRNA expression}

The concentration of $\mathrm{Hb}$ in the Han population that possessed the homozygotic plateau-adaptive 
240

241

242

243

244

245

246

247

248

249

250

251

252

253

254

255

256

257

258

259

260

261

262

263

264

265

266

genotype involving three SNPs of the EPAS1 gene was reduced, but the difference was not statistically significant; thus, it was unclear whether or not the Hb level is affected by gene expression. We therefore grouped the subjects into wild-type, plateau-adaptive heterozygous type, and plateau-adaptive homozygous type and measured the EPAS1 gene expression levels in plains male subjects before and after acute entry into the plateau. The results showed that the EPASI gene expression level of individuals who possessed the "A-A" homozygotic genotype at the rs 13419896 locus and were living in the plains was lower than that of individuals with the wild-type and heterozygotic genotype, whereas the EPAS1 gene expression in the "A-A" plateau-adaptive homozygous genotype group was higher than that in the wild-type and plateau-adaptive heterozygous population after entering the plateau (Fig. 3).

\section{Differences in Raman spectra between the Tibetan and Han groups}

Our previous study has demonstrated significant differences in the $\mathrm{P}_{50}$ values of $\mathrm{Hb}$ and oxygen affinity in different subgroups( $\mathrm{Li}$ et al. 2018), suggesting that, in addition to the changes in $\mathrm{Hb}$ concentration observed in the above studies, $\mathrm{Hb}$ may undergone conformational changes that are conducive to plateau acclimation. Therefore, Raman spectroscopy was used to perform $\mathrm{Hb}$ conformational analysis. Compared with the plains Han group, the Han group acutely entering the plateau showed a Raman band at $1341 \mathrm{~cm}^{-1}$; this band disappeared after 30 days of plateau acclimation, accompanied by the appearance of a Raman band at $1300 \mathrm{~cm}^{-1}$. The intensity of the Raman peak at $1375 \mathrm{~cm}^{-1}$ increased with duration of residence in the plateau, but it decreased after 30 days of plateau acclimation, accompanied by the appearance of the Raman band at $1355 \mathrm{~cm}^{-1}$. The group living in the plateau for a long time only displayed a characteristic peak at $1375 \mathrm{~cm}^{-1}$, and the peak intensity was reduced. The intensity of the Raman peak at $1546 \mathrm{~cm}^{-1}$ increased with time after entry into the plateau, reaching a maximum at 30 days of plateau acclimation; it was weakened in the group living in the plateau for a long time. The intensity of the Raman peak at $1585 \mathrm{~cm}^{-1}$ increased with time after entry into the plateau and decreased after 30 days of plateau acclimation. The group living in the plateau for a long time displayed a characteristic peak at 1585 $\mathrm{cm}^{-1}$, and the intensity of this peak was even weaker. The Raman intensity peak at $1638 \mathrm{~cm}^{-1}$ 
267 increased with duration after entry into the plateau, began to decrease at 30 days of plateau 268 acclimation and was even weaker in the group that had lived in the plateau for a long time (Fig. $2694)$.

270 The Raman spectrum of RBCs obtained from the Tibetan population contained two bands that 271 differed from those found in the plains group. The Raman results for six samples (60\%) showed 272 that the Raman peak intensity of the bands at $1375 \mathrm{~cm}^{-1}, 1585$, and $1638 \mathrm{~cm}^{-1}$ were basically the 273 same in Tibetans as in the plains group, but new Raman bands appeared at $1355 \mathrm{~cm}^{-1}, 1546$, and $2741603 \mathrm{~cm}^{-1}$. The Raman results for four samples $(40 \%)$ were similar to those of the plains group, 275 but the intensity of the peak was enhanced (Fig. 4).

\section{Discussion}

277 The unique natural environment of the high-altitude plateau has a great influence on human 278 physiology, ability to work and physical and mental health. Physiological changes, such as 279 significant increases in red blood cell counts and $\mathrm{Hb}$ level can improve the oxygen supply to 280 tissues, allowing individuals to quickly adapt to the plateau. For the first time, this study conducted research on the acclimation of the Han population living in the plateau for different periods of

282 time. The levels of $\mathrm{Hb}$ and $\mathrm{S}_{\mathrm{P}} \mathrm{O}_{2}$ in the different population and the relationships with the gene polymorphisms and mRNA expression of the hypoxia-induction-related EPAS1 were analyzed.

We also detected differences in the structure and function of $\mathrm{Hb}$ in the different subgroups that can be used as a means of identifying Han populations that are able to adapt to the plateau. The results of this study showed that the $\mathrm{Hb}$ level of plains Han people increased significantly three days after they entered the plateau and was maintained at an average level of $176 \mathrm{~g} / \mathrm{L}$. Thus, the increased $\mathrm{Hb}$ levels could supply adequate oxygen to organs in the hypoxic environment, which is an important mechanism of altitude acclimation (Chen et al. 2014). In the plateau hypoxic environment, $\mathrm{S}_{\mathrm{P}} \mathrm{O}_{2}$ values were significantly reduced in plains Han group; this suggests that the change in $\mathrm{Hb}$ content and conformation may lead to differences in physiological indicators among different populations in the high altitude environment. 
294 hypoxic conditions, EPAS1 activates the transcription of its downstream target gene $E P O$, thus

295

296

297

298

299

300

301

302

303

304

305

306

307

308

309

310

311

312

313

314

315

316

317

318

319

320

increasing the number of red blood cell to meet the body's oxygen supply and allowing adaptation to the plateau(Peng et al. 2017). Studies have shown that EPAS1 gene expression is also associated with EPO levels (Lee \& Percy 2011; Percy et al. 2008). In a hypoxic environment, HIF-2 $\alpha$, encoded by EPAS1, may accumulate in cytoplasm and then translocate into the nucleus and bind to the hypoxia response element to activate the expression of downstream genes, sunch as $E P O$ (Petousi \& Robbins 2014) and $V E G F$ (Sergi 2019). Beall et al.(Beall et al. 2010) found that 31 SNPs in the EPAS1 gene of the Tibetan population showed linkage disequilibrium and were related to the $\mathrm{Hb}$ concentration and that the average $\mathrm{Hb}$ concentration of homozygous carriers of the allele mutation was $8 \mathrm{~g} / \mathrm{L}$ lower than that in heterozygous carriers. Peng et al.(Peng et al. 2011) found that the $\mathrm{Hb}$ concentrations of Tibetans carrying the plateau-adaptive EPAS1 rs149594770 and rs73926265 locus genotypes were decreased by $6.15 \mathrm{~g} / \mathrm{L}$ and $12.65 \mathrm{~g} / \mathrm{L}$, respectively.

This study also analyzed the causes of the changes in $\mathrm{Hb}$ and $\mathrm{S}_{\mathrm{P}} \mathrm{O}_{2}$ levels in different subgroups from the perspective of EPAS1 gene polymorphism, and mRNA expression. First, the EPAS1 gene polymorphism study showed that the frequency of the rs13419896 A allele in the Tibetan population was $87.0 \%$, significantly higher than its frequency in the plains population $(31.5 \%)$ $(\mathrm{P}<0.001)$; the frequency of the $\mathrm{rs} 1868092 \mathrm{~A}$ allele in the Tibetan population was $76.5 \%$, significantly higher than that of in the plains population $(11.0 \%)(\mathrm{P}<0.001)$, and the frequency of the rs4953354 $\mathrm{G}$ allele was significantly higher in the Tibetan population than in the plains population $(81.0 \%$ vs $13.5 \%)(\mathrm{P}<0.001)$. Therefore, we believe that these three SNPs have important roles in plateau adaptation and plateau acclimation. Our analysis also showed that individuals in Tibetan and Han populations with the plateau-adaptive genotypes of three SNPs had lower $\mathrm{Hb}$ levels than individuals of the other two genotypes.

Second, the EPASI expression levels in the plains Han population were found to increase after this population acutely entered the plateau and to then decrease after acclimation. In addition, the EPAS1 expression level in the Han group who had lived in the plateau for more than 10 years was significantly higher than that in the plains group. Stimulation of the expression of hypoxia- 
321 inducible factor EPAS1 by the plateau hypoxic environment is a manifestation of plateau 322 acclimation.

323 To clarify whether genetic mutations in the plains population affect expression of the EPAS1 gene, 324 we also performed a comparison of genotypes and $\mathrm{Hb}$ levels in different groups. The results 325 showed that in the plains group, EPAS1 expression was significantly lower in individuals carrying 326 the "A-A" homozygous plateau-adaptive genotype of the rs13419896 locus than individuals 327 carrying the "G-G/A-G" genotypes. After the subjects had remained in the plateau for 3-7 days, 328 gene expression was higher in homozygous mutants than the "G-G" wild-type and "A-G" 329 heterozygous populations. However, there was no significant difference between different 330 rs1868092 and rs4953354 genotypes before and after entering the plateau. This finding indicates 331 that the Han population carrying the AA genotype of the rs13419896 locus expresses more $\mathrm{Hb}$ 332 after entering the plateau. However, our test results showed that Hb levels in people with AA genotypes were lower than $\mathrm{Hb}$ levels in people with "G-G" or "A-G" genotypes and were not consistent with gene expression levels. We speculate that the expression of $\mathrm{Hb}$ under hypoxic conditions was affected by factors other than the EPAS1 polymorphism and that the single-site mutation in the gene was also not sufficient to affect the expression level of the gene.

Our previous study of $\mathrm{Hb}$ and oxygen affinity $\left(\mathrm{P}_{50}\right)$ revealed significant differences in different among populations. In this study, Raman spectroscopy was used to investigate the $\mathrm{Hb}$ conformation. The region from $1340-1390 \mathrm{~cm}^{-1}$ is an area that is sensitive to the density of electrons in the heme ring; it is also a marker band for the redox state of heme(Kitagawa et al. 1978). The increased density at the initial stage of entering the plateau may be the result of excessive release of oxygen caused by an increase in $\mathrm{P}_{50}$ and subsequent oxidation of heme. The spectrum in the $1350-1380 \mathrm{~cm}^{-1}$ region reflects changes in the quaternary structure of $\mathrm{Hb}$ (Rousseau et al. 1980). Changes in this region suggest that the stability of $\mathrm{Hb}$ is destroyed. The $\mathrm{P}_{50}$ value increased in the early stage of acute plateau adaptation (the $1375 \mathrm{~cm}^{-1}$ band was enhanced); after 30 days of acclimation, the $\mathrm{P}_{50}$ value was significantly lower than the normal value of the plains population (the $1355 \mathrm{~cm}^{-1}$ band appeared)(Li et al. 2018). In individuals who had lived in the 
348 plateau for a long time, only the $1375 \mathrm{~cm}^{-1}$ band showed decreased intensity, this may may initiate 349 the regulation mechanism post-compensation and regulate the $\mathrm{P}_{50}$ changes. The region of 1240$3501300 \mathrm{~cm}^{-1}$ is related to the secondary structure of $\mathrm{Hb}$ (Wood et al. 2007). After 30 days of plateau 351 acclimation, bands appeared at $1300 \mathrm{~cm}^{-1}$, possible as a result of changes in $\mathrm{Hb}$ secondary

352

353

354

structure. The hemoglobin pockets in the globin chains consist of connected $\alpha$-helices, and the changes may indicate changes in hemoglobin tertiary structure and spatial conformation after altitude adaptation. The bands at 1585 and $1638 \mathrm{~cm}^{-1}$ are related to oxyhemoglobin(Lu et al. 2014). The Raman band changes at 3-7 days after entering the plateau. The corresponding detection result shows that $\mathrm{P}_{50}$ is increased and the organism is likely to release oxygen. However, after 30 days of high altitude acclimation and after long years of living in the plateau, the value is reduced. It is possible that the body may adjust the structure of the hemoglobin to adapt to the high altitude environment. At this time, the $\mathrm{P}_{50}$ result is reduced so that oxygen can be used for local tissue with extreme hypoxia(Wood et al. 2001). High spin state characteristic peaks of the heme near 1546 and $1603 \mathrm{~cm}^{-1}$, suggesting that the ions of $\mathrm{Hb}$ tend to show a high spin state after 30 days of altitude adaptation. In addition, the intensities of each characteristic Hb peak are weakened in Han people living in the plateau for long periods of time; this may indicate that the internal environment of the red blood cells of these individuals has undergone specific changes that result in the weakening of the characteristic peaks of hemoglobin. However, its specific mechanism of this effect will require studied.

Approximately $60 \%$ of the Tibetan population is similar to the Han people who have lived in the plateau for a long time. The intensity of the Raman peak at $1375 \mathrm{~cm}^{-1}$ is lower in Tibetan than in the plains group, and a Raman peak appears at $1355 \mathrm{~cm}^{-1}$. The appearance of a Raman peak in this region indicates that the changes in the structure of $\mathrm{Hb}$ are associated with changes in oxygen affinity. The bands at 1585 and $1638 \mathrm{~cm}^{-1}$ are related to oxyhemoglobin. The reduction in the intensities of the peaks in this region in the Tibetan population may be due to the adjustment of the hemoglobin structure to adapt to the high altitude environment and ultimately provide better adapt to the hypoxic environment. The corresponding reduction in $\mathrm{P}_{50}$ ensures that oxygen can be 
375

376

377

378

379

380

381

382

383

384

385

386

387

388

389

390

391

392

393

394

395

396

397

398

399

400

401

402

403

404

405

406

used for the hypoxic tissue. The 1546 and $1603 \mathrm{~cm}^{-1}$ peaks indicates that iron in Tibetan hemoglobin tends to show a high spin state, resulting in a decrease in $\mathrm{P}_{50}$. The Tibetan population has lived in a plateau environment for generations and their hemoglobin structure must be better adapted to hypoxia, but its specific adaptation mechanism should still be further explored.

\section{Conclusion}

In summary, under plateau hypoxic conditions, the Han population of the plains may rapidly acclimate to hypoxia through the EPAS1 gene polymorphism and increase EPAS1 mRNA expression and even changes in the hemoglobin conformation.

\section{Declarations}

\section{Acknowledgements}

The authors thank Ying Wang for helpful discussion and review of this manuscript.

\section{Reference}

Beall CM, Cavalleri GL, Deng L, Elston RC, Gao Y, Knight J, Li C, Li JC, Liang Y, McCormack M, Montgomery HE, Pan H, Robbins PA, Shianna KV, Tam SC, Tsering N, Veeramah KR, Wang W, Wangdui P, Weale ME, Xu Y, Xu Z, Yang L, Zaman MJ, Zeng C, Zhang L, Zhang X, Zhaxi P, and Zheng YT. 2010. Natural selection on EPAS1 (HIF2alpha) associated with low hemoglobin concentration in Tibetan highlanders. Proc Natl Acad Sci U S A 107:11459-11464. 10.1073/pnas.1002443107

Beall CM, Decker MJ, Brittenham GM, Kushner I, Gebremedhin A, and Strohl KP. 2002. An Ethiopian pattern of human adaptation to high-altitude hypoxia. Proc Natl Acad Sci U S A 99:17215-17218. 10.1073/pnas.252649199

Bigham AW, and Lee FS. 2014. Human high-altitude adaptation: forward genetics meets the HIF pathway. Genes Dev 28:2189-2204. 10.1101/gad.250167.114

Chen Y, Jiang C, Luo Y, Liu F, and Gao Y. 2014. An EPAS1 haplotype is associated with high altitude polycythemia in male Han Chinese at the Qinghai-Tibetan plateau. Wilderness Environ Med 25:392-400. 10.1016/j.wem.2014.06.003

Hu CJ, Wang LY, Chodosh LA, Keith B, and Simon MC. 2003. Differential roles of hypoxia-inducible factor 1alpha (HIF-1alpha) and HIF-2alpha in hypoxic gene regulation. Mol Cell Biol 23:9361-9374.

Kitagawa T, Ozaki Y, and Kyogoku Y. 1978. Resonance Raman studies on the ligand-iron interactions in hemoproteins and metallo-porphyrins. Adv Biophys 11:153-196.

Lee FS, and Percy MJ. 2011. The HIF pathway and erythrocytosis. Annu Rev Pathol 6:165-192. 10.1146/annurevpathol-011110-130321 
407

408

409

410

411

412

413

414

415

416

417

418

419

420

421

422

423

424

425

426

427

428

429

430

431

432

433

434

435

436

437

438

439

440

441

442

443

444

445

446

447

Lendahl U, Lee KL, Yang H, and Poellinger L. 2009. Generating specificity and diversity in the transcriptional response to hypoxia. Nat Rev Genet 10:821-832. 10.1038/nrg2665

Li C, Li X, Liu J, Fan X, You G, Zhao L, Zhou H, Li J, and Lei H. 2018. Investigation of the differences between the Tibetan and Han populations in the hemoglobin-oxygen affinity of red blood cells and in the adaptation to high-altitude environments. Hematology 23:309-313. 10.1080/10245332.2017.1396046

Lu M, Zhao L, Wang Y, You G, Kan X, Zhang Y, Zhang N, Wang B, Guo YJ, and Zhou H. 2014. Measurement of the methemoglobin concentration using Raman spectroscopy. Artif Cells Nanomed Biotechnol 42:63-69. $10.3109 / 21691401.2013 .775577$

Meier D, Collet TH, Locatelli I, Cornuz J, Kayser B, Simel DL, and Sartori C. 2017. Does This Patient Have Acute Mountain Sickness?: The Rational Clinical Examination Systematic Review. JAMA 318:1810-1819. 10.1001/jama.2017.16192

Peng Y, Cui C, He Y, Ouzhuluobu, Zhang H, Yang D, Zhang Q, Bianbazhuoma, Yang L, He Y, Xiang K, Zhang X, Bhandari S, Shi P, Yangla, Dejiquzong, Baimakangzhuo, Duojizhuoma, Pan Y, Cirenyangji, Baimayangji, Gonggalanzi, Bai C, Bianba, Basang, Ciwangsangbu, Xu S, Chen H, Liu S, Wu T, Qi X, and Su B. 2017. Down-Regulation of EPAS1 Transcription and Genetic Adaptation of Tibetans to High-Altitude Hypoxia. Mol Biol Evol 34:818-830. 10.1093/molbev/msw280

Peng Y, Yang Z, Zhang H, Cui C, Qi X, Luo X, Tao X, Wu T, Ouzhuluobu, Basang, Ciwangsangbu, Danzengduojie, Chen H, Shi H, and Su B. 2011. Genetic variations in Tibetan populations and high-altitude adaptation at the Himalayas. Mol Biol Evol 28:1075-1081. 10.1093/molbev/msq290

Percy MJ, Furlow PW, Lucas GS, Li X, Lappin TR, McMullin MF, and Lee FS. 2008. A gain-of-function mutation in the HIF2A gene in familial erythrocytosis. N Engl J Med 358:162-168. 10.1056/NEJMoa073123

Petousi N, Croft QP, Cavalleri GL, Cheng HY, Formenti F, Ishida K, Lunn D, McCormack M, Shianna KV, Talbot NP, Ratcliffe PJ, and Robbins PA. 2014. Tibetans living at sea level have a hyporesponsive hypoxia-inducible factor system and blunted physiological responses to hypoxia. J Appl Physiol (1985) 116:893-904. 10.1152/japplphysiol.00535.2013

Petousi N, and Robbins PA. 2014. Human adaptation to the hypoxia of high altitude: the Tibetan paradigm from the pregenomic to the postgenomic era. J Appl Physiol (1985) 116:875-884. 10.1152/japplphysiol.00605.2013

Rousseau DL, Shelnutt JA, Henry ER, and Simon SR. 1980. Raman difference spectroscopy of tertiary and quaternary structure changes in methaemoglobins. Nature 285:49-51.

Sergi C. 2019. EPAS 1, congenital heart disease, and high altitude: disclosures by genetics, bioinformatics, and experimental embryology. Biosci Rep 39. 10.1042/BSR20182197

Simonson TS, Huff CD, Witherspoon DJ, Prchal JT, and Jorde LB. 2015. Adaptive genetic changes related to haemoglobin concentration in native high-altitude Tibetans. Exp Physiol 100:1263-1268. 10.1113/EP085035

Wood BR, Caspers P, Puppels GJ, Pandiancherri S, and McNaughton D. 2007. Resonance Raman spectroscopy of red blood cells using near-infrared laser excitation. Anal Bioanal Chem 387:1691-1703. 10.1007/s00216-0060881-8

Wood BR, Tait B, and McNaughton D. 2001. Micro-Raman characterisation of the R to $\mathrm{T}$ state transition of haemoglobin within a single living erythrocyte. Biochim Biophys Acta 1539:58-70.

Xu J, Yang YZ, Tang F, Ga Q, Tana W, and Ge RL. 2015. EPAS1 Gene Polymorphisms Are Associated With High Altitude Polycythemia in Tibetans at the Qinghai-Tibetan Plateau. Wilderness Environ Med 26:288-294. 10.1016/j.wem.2015.01.002

Peer) reviewing PDF | (2019:07:39345:1:1:NEW 7 Sep 2019) 
448

449 
450 Figure legends:

451 Fig1. The differences of physiological indexes between Tibetan and Han ethnic groups in the plateau 452 hypoxic environment.(A) The hemoglobin level in different population.(B) The hemoglobin level in 453 different population. ${ }^{*} P<0.05$ or $* * * P<0.001$ for indicated group versus plain group. n.s: No statistical 454 difference.

455

456 Fig2. The differences in EPAS1 mRNA expression between the Tibetan and Han populations. mRNA was 457 extracted from peripheral blood in different group and reversed into cDNA, and RPL13A was used to 458 calibrate sample loading. $* P<0.05$ or $* * * P<0.001$ for indicated group versus plain group. n.s: No statistical 459 difference.

460

461 Fig3. Expression level of different genotypes in EPSA1 gene at Han plateau after Entering Plateau. 462 Stratified analysis based on different genotypes was used to analyze the expression levels of EPAS1 gene.

463

464 Fig4. Raman spectra of RBC in Tibetans and plain population. Comparison of the spectra recorded for the 465 different group (A) Han population before or after entering plateau (B) Tibetan using 514nm laser excitation 466 wavelengths, showing the major band assignments in the range of $500-2000 \mathrm{~cm}^{-1}$. 
Figure 1

The differences of physiological indexes between Tibetan and Han ethnic groups in the plateau hypoxic environment.

(A) The hemoglobin level in different population.(B) The hemoglobin level in different population. $* P<0.05$ or ${ }^{* * * P}<0.001$ for indicated group versus plain group. N.S: No statistical difference.

A

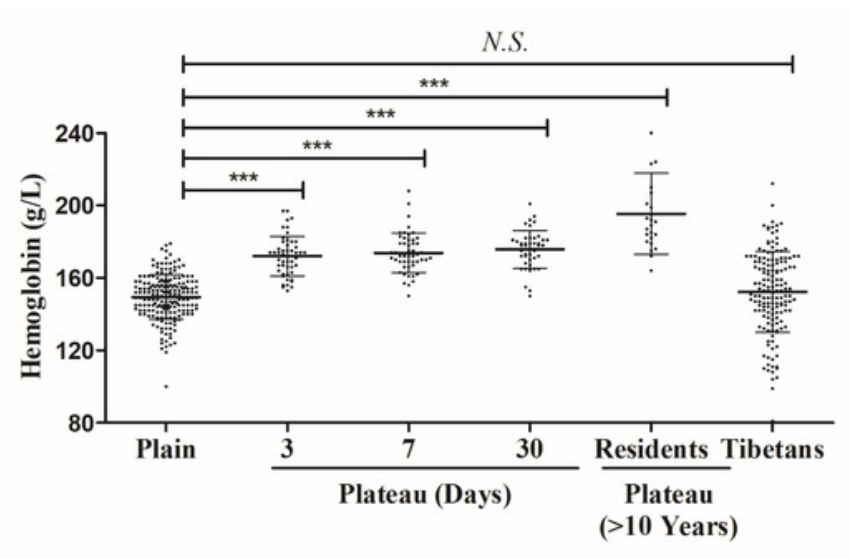

B

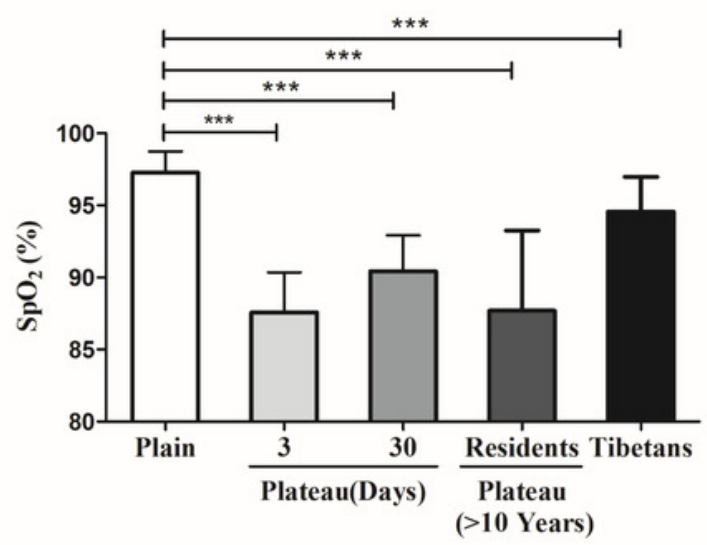


Figure 2

The differences in EPAS1 mRNA expression between the Tibetan and Han populations.

mRNA was extracted from peripheral blood in different group and reversed into cDNA, and RPL13A was used to calibrate sample loading. $* P<0.05$ or $* * * P<0.001$ for indicated group versus plain group. N.S: No statistical difference.

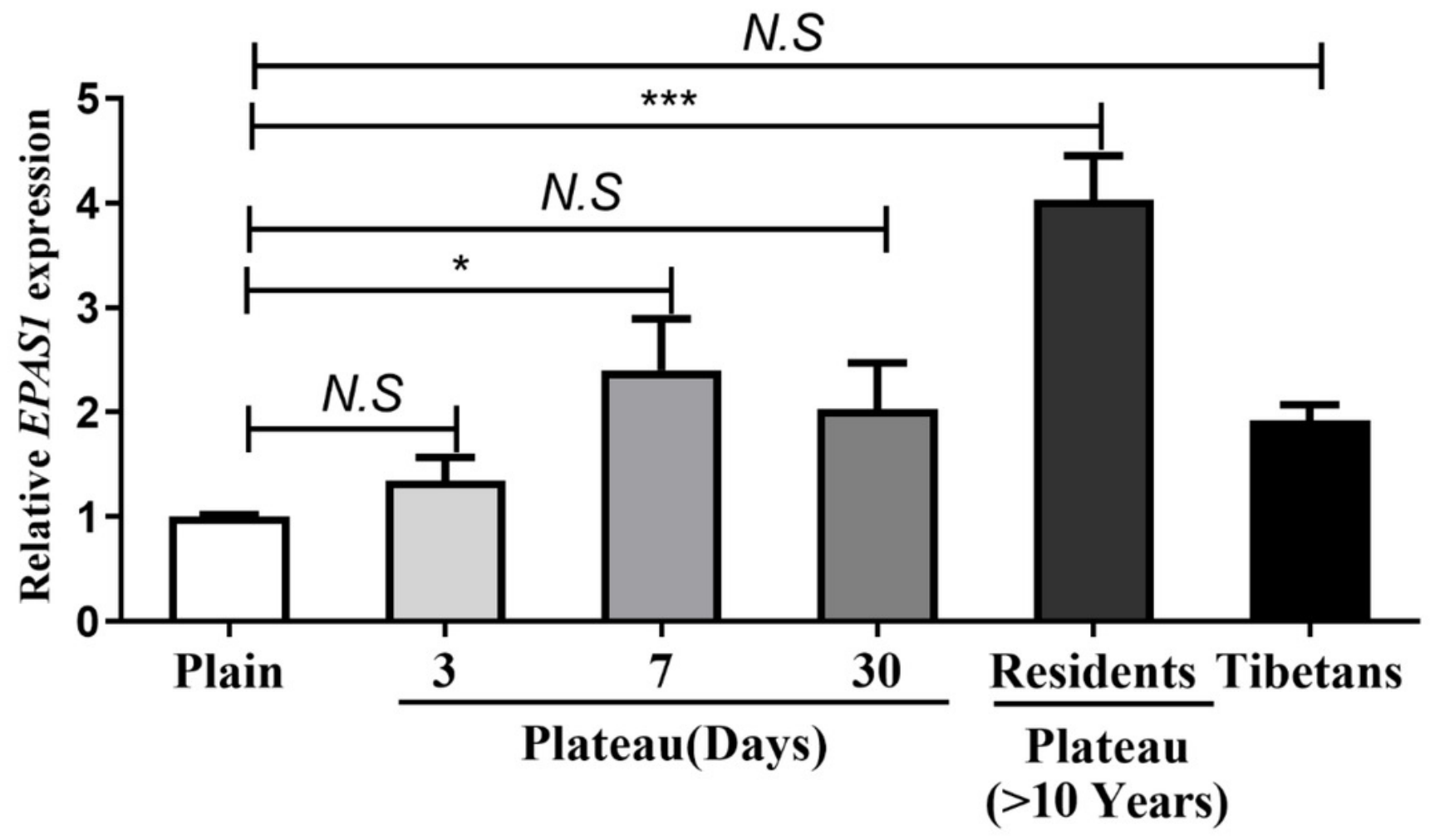


Figure 3

Expression level of different genotypes in EPSA1 gene at Han plateau after Entering Plateau.

Stratified analysis based on different genotypes was used to analyze the expression levels of EPAS1 gene.

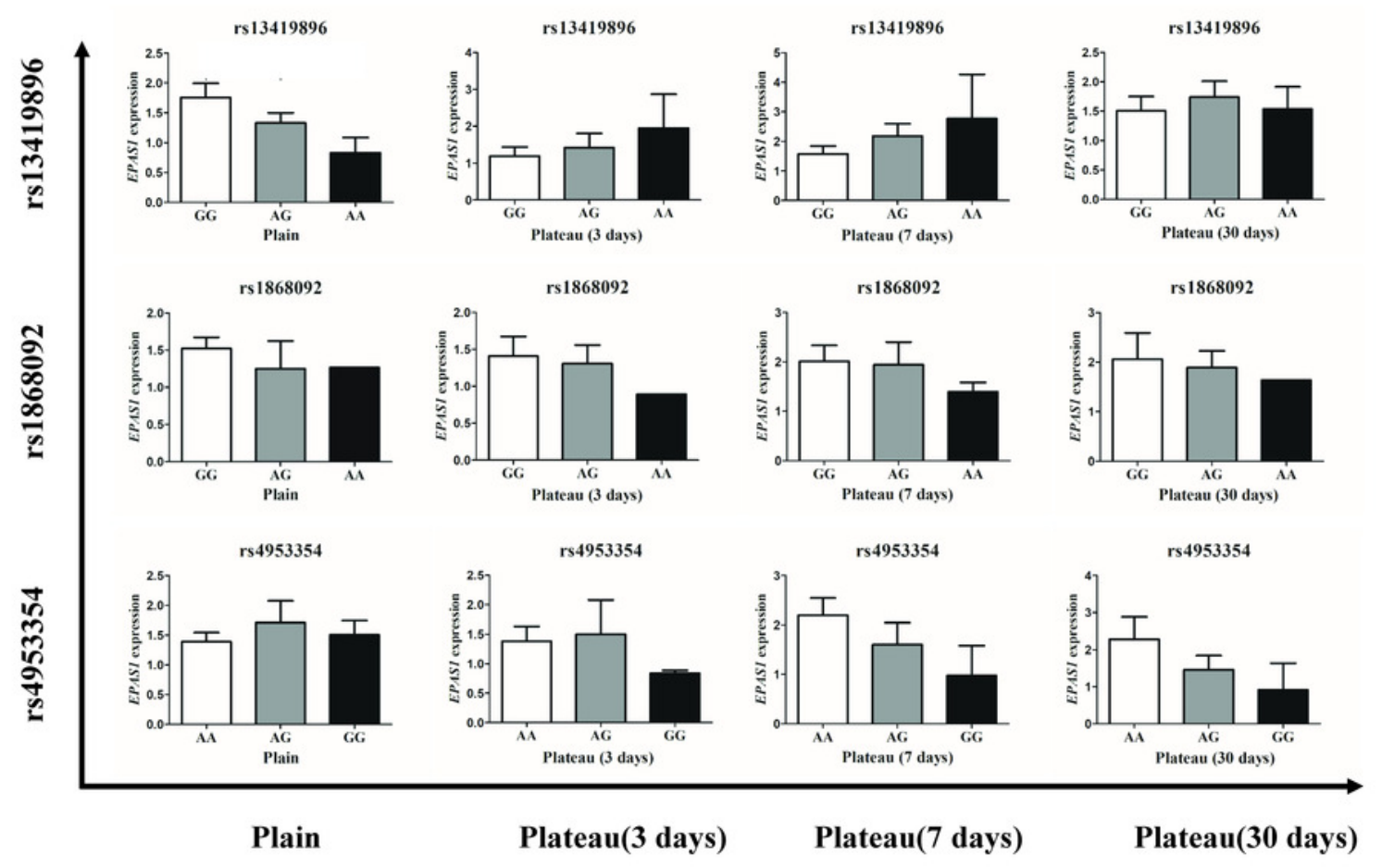


Figure 4

Raman spectra of RBC in Tibetans and plain population.

Comparison of the spectra recorded for the different group (A) Han population before or after entering plateau (B) Tibetan using 514nm laser excitation wavelengths, showing the major band assignments in the range of $500-2000 \mathrm{~cm}^{-1}$.

A

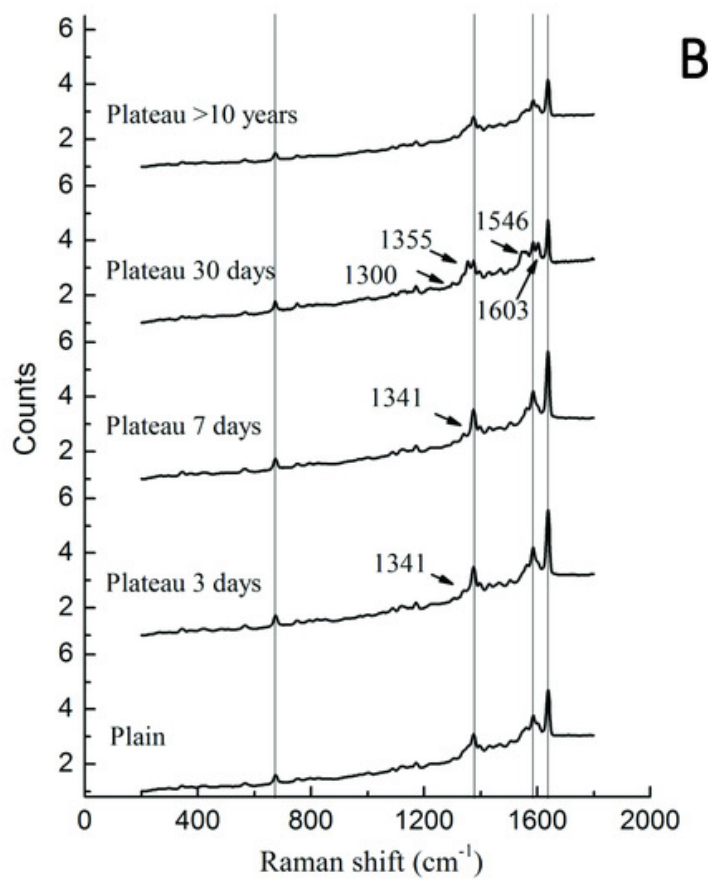

B

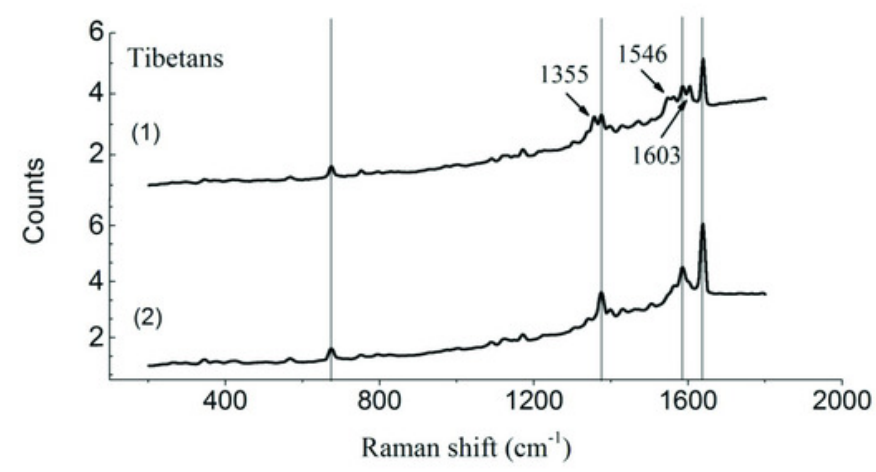




\section{Table 1 (on next page)}

The baseline characteristics of each group. 
1 Table 1. The baseline characteristics of each group

\begin{tabular}{|c|c|c|c|c|c|c|}
\hline & No. & Age & BMI & Smoking & Altitude(m) & First exposure? \\
\hline Plains & 190 & $24.12 \pm 4.40$ & $23.00 \pm 1.12$ & No & 500 & - \\
\hline $\begin{array}{l}\text { Han entered plateau } \\
3^{\text {rd }} \text { day }\end{array}$ & 50 & $23.50 \pm 4.42$ & $23.07 \pm 1.16$ & No & 4200 & $\checkmark$ \\
\hline $\begin{array}{l}\text { Han entered plateau } \\
7^{\text {th }} \text { day }\end{array}$ & 50 & $19.48 \pm 1.25$ & $22.29 \pm 1.35$ & No & 4200 & $\checkmark$ \\
\hline $\begin{array}{l}\text { Han entered plateau } \\
30^{\text {th }} \text { day }\end{array}$ & 44 & $22.70 \pm 3.60$ & $22.25 \pm 1.50$ & No & 3700 & $\checkmark$ \\
\hline $\begin{array}{l}\text { The plateau Han } \\
\text { population (living in } \\
\text { plateau>10years) }\end{array}$ & 24 & $41.50 \pm 7.60$ & $22.63 \pm 1.39$ & No & 3700 & - \\
\hline Tibetan & 155 & $35.31 \pm 15.53$ & $23.16 \pm 1.76$ & No & 3700 & - \\
\hline
\end{tabular}


Table 2 (on next page)

Three SNPs genotype and allele frequency distribution of EPAS1 gene in different populations. 
1 Table 2. Three SNPs genotype and allele frequency distribution of EPAS1 gene in different 2 populations

\begin{tabular}{|c|c|c|c|c|c|}
\hline SNP & $\begin{array}{c}\text { Genotype or allele } \\
\text { associated with SNP }\end{array}$ & $\begin{array}{c}\text { Native Tibetans } \\
(\mathrm{n} \% . \mathrm{N}=155)\end{array}$ & $\begin{array}{l}\text { Plain group } \\
(\mathrm{n} \%, \mathrm{~N}=190)\end{array}$ & OR $(95 \% \mathrm{CI})$ & $P$ value ${ }^{*}$ \\
\hline \multicolumn{6}{|l|}{ rs13419896 } \\
\hline \multirow[t]{3}{*}{ Genotype } & $\mathrm{G} / \mathrm{G}$ & $4(2.6)$ & $83(43.7)$ & 1 & \\
\hline & $\mathrm{G} / \mathrm{A}$ & $35(22.6)$ & $90(47.4)$ & $0.124(0.042-0.364)$ & 0.000 \\
\hline & $\mathrm{A} / \mathrm{A}$ & $116(74.8)$ & $17(8.9)$ & $0.007(0.002-0.022)$ & 0.000 \\
\hline \multirow[t]{2}{*}{ Allele } & G & $43(13.9)$ & $256(67.4)$ & 1 & \\
\hline & A & $267(86.1)$ & $124(32.6)$ & $0.078(0.053-0.115)$ & 0.000 \\
\hline \multicolumn{6}{|l|}{ rs1868092 } \\
\hline \multirow[t]{3}{*}{ Genotype } & $\mathrm{G} / \mathrm{G}$ & $12(7.7)$ & $152(80.0)$ & 1 & \\
\hline & $\mathrm{G} / \mathrm{A}$ & $61(39.4)$ & $37(19.5)$ & $0.048(0.023-0.098)$ & 0.000 \\
\hline & $\mathrm{A} / \mathrm{A}$ & $82(52.9)$ & $1(0.5)$ & $0.001(0.000-0.008)$ & 0.000 \\
\hline \multirow[t]{2}{*}{ Allele } & G & $85(27.4)$ & $341(89.7)$ & 1 & \\
\hline & $\mathrm{A}$ & $225(72.6)$ & $39(10.3)$ & $0.043(0.029-0.065)$ & 0.000 \\
\hline \multicolumn{6}{|l|}{ rs4953354 } \\
\hline \multirow[t]{3}{*}{ Genotype } & $\mathrm{A} / \mathrm{A}$ & $18(11.6)$ & $144(75.8)$ & 1 & \\
\hline & $\mathrm{G} / \mathrm{A}$ & $40(25.8)$ & $44(23.1)$ & $0.138(0.072-0.264)$ & 0.000 \\
\hline & $\mathrm{G} / \mathrm{G}$ & $97(62.6)$ & $2(10.1)$ & $0.003(0.001-0.011)$ & 0.000 \\
\hline \multirow[t]{2}{*}{ Allele } & A & $76(24.5)$ & $332(87.4)$ & 1 & \\
\hline & G & $234(75.5)$ & $48(12.6)$ & $0.047(0.032-0.070)$ & 0.000 \\
\hline
\end{tabular}

3 NOTE: OR: Odds ratio, $\mathrm{OR}>1$ indicates that this factor is a risk factor; OR value $<1$ indicates that this factor is a protective factor;

$4 *$ means Heterozygous mutation or Homozygous mutation group compared with wild type group, $\mathrm{P}<0.05$ was considered to be 5 statistically significant.

6 


\section{Table 3(on next page)}

Genotype of three SNPs within the EPAS1 gene and differences in Hb levels in different populations. 
1 Table3. Genotype of three SNPs within the EPAS1 gene and differences in Hb levels in

2 different populations

\begin{tabular}{|c|c|c|c|c|}
\hline & $\mathrm{SNP}_{\text {Sites }}^{\mathrm{Hb}(\mathrm{g} / \mathrm{L})}$ & Wild type & $\begin{array}{c}\text { Heterozygous } \\
\text { mutation }\end{array}$ & $\begin{array}{c}\text { Homozygous } \\
\text { mutation }\end{array}$ \\
\hline \multirow{3}{*}{$\begin{array}{l}\text { Plain } \\
\text { group }\end{array}$} & rs13419896 & $151.2 \pm 12.2$ & $147.6 \pm 12.0$ & $149.1 \pm 9.8$ \\
\hline & rs1868092 & $149.8 \pm 12.3$ & $147.5 \pm 10.3$ & $133.0 \pm 0.0$ \\
\hline & rs4953354 & $150.1 \pm 11.2$ & $148.0 \pm 14.0$ & $140.7 \pm 12.4$ \\
\hline \multirow{3}{*}{$\begin{array}{l}\text { Plateau } \\
\text { (3 days) }\end{array}$} & rs13419896 & $173.7 \pm 12.9$ & $171.6 \pm 10.5$ & $170.4 \pm 5.3$ \\
\hline & rs1868092 & $172.6 \pm 11.4$ & $172.3 \pm 10.1$ & 164.0 \\
\hline & rs4953354 & $172.4 \pm 10.1$ & $172.3 \pm 14.4$ & $172.5 \pm 12.0$ \\
\hline \multirow{3}{*}{$\begin{array}{l}\text { Plateau } \\
\text { (7 days) }\end{array}$} & rs13419896 & $174.9 \pm 9.4$ & $174.3 \pm 12.7$ & $169.7 \pm 10.4$ \\
\hline & rs1868092 & $170.5 \pm 28.9$ & $173.8 \pm 10.7$ & 150.0 \\
\hline & rs4953354 & $169.3 \pm 30.1$ & $175.8 \pm 10.6$ & $166.0 \pm 22.6$ \\
\hline \multirow{3}{*}{$\begin{array}{c}\text { Plateau } \\
\text { (30 days) }\end{array}$} & rs13419896 & $177.7 \pm 10.9$ & $175.7 \pm 9.5$ & $170.8 \pm 11.7$ \\
\hline & rs1868092 & $177.7 \pm 8.7$ & $169.4 \pm 14.8$ & 165.0 \\
\hline & rs4953354 & $176.1 \pm 7.4$ & $175.9 \pm 17.0$ & $173.0 \pm 11.3$ \\
\hline \multirow{3}{*}{$\begin{array}{c}\text { Plateau } \\
(>10 \text { years })\end{array}$} & rs13419896 & $174.5 \pm 11.3$ & $171.1 \pm 18.8$ & $158.2 \pm 19.8$ \\
\hline & rs1868092 & $171.2 \pm 16.3$ & $171.3 \pm 19.5$ & $170.0 \pm 6.0$ \\
\hline & rs4953354 & $170.3 \pm 16.1$ & $169.4 \pm 19.0$ & $177.4 \pm 11.0$ \\
\hline \multirow{3}{*}{$\begin{array}{l}\text { Native } \\
\text { Tibetans }\end{array}$} & rs13419896 & $172.0 \pm 16.8$ & $158.9 \pm 31.5$ & $153.8 \pm 27.7$ \\
\hline & rs1868092 & $189.7 \pm 18.0$ & $154.7 \pm 29.4$ & $150.1 \pm 25.1$ \\
\hline & rs4953354 & $160.7 \pm 24.3$ & $158.3 \pm 32.1$ & $152.9 \pm 28.7$ \\
\hline
\end{tabular}

3 


\section{Table 4 (on next page)}

Genotype of three SNPs within the EPAS1 gene and differences in $\mathrm{SpO}_{2}$ value in different populations. 
1 Table4. Genotype of three SNPs within the $E P A S 1$ gene and differences in $\mathrm{SpO}_{2}$ value in

2 different populations

\begin{tabular}{|c|c|c|c|c|}
\hline & $\mathrm{SpO}_{2}(\%)$ & Wild type & $\begin{array}{c}\text { Heterozygous } \\
\text { mutation }\end{array}$ & $\begin{array}{c}\text { Homozygous } \\
\text { mutation }\end{array}$ \\
\hline \multirow{3}{*}{$\begin{array}{l}\text { Plain } \\
\text { group }\end{array}$} & rs13419896 & $97.30 \pm 1.36$ & $97.08 \pm 1.60$ & $99.11 \pm 1.64$ \\
\hline & rs1868092 & $97.33 \pm 1.37$ & $97.00 \pm 2.00$ & $95.69 \pm 3.56$ \\
\hline & rs4953354 & $97.02 \pm 1.53$ & $97.92 \pm 1.31$ & $98.55 \pm 1.41$ \\
\hline \multirow{3}{*}{$\begin{array}{l}\text { Plateau } \\
\text { (3 days) }\end{array}$} & rs13419896 & $87.44 \pm 3.37$ & $87.81 \pm 3.29$ & $87.62 \pm 1.60$ \\
\hline & rs1868092 & $87.85 \pm 3.16$ & $86.64 \pm 2.84$ & -- \\
\hline & rs 4953354 & $87.76 \pm 3.05$ & $87.15 \pm 3.41$ & -- \\
\hline \multirow{3}{*}{$\begin{array}{c}\text { Plateau } \\
\text { (30 days) }\end{array}$} & rs13419896 & $90.40 \pm 2.46$ & $90.18 \pm 2.96$ & $91.81 \pm 1.41$ \\
\hline & rs1868092 & $90.06 \pm 2.62$ & $91.86 \pm 1.77$ & -- \\
\hline & rs 4953354 & $90.31 \pm 2.84$ & $90.56 \pm 1.24$ & -- \\
\hline \multirow{3}{*}{$\begin{array}{c}\text { Plateau } \\
(>10 \text { years })\end{array}$} & rs13419896 & $88.75 \pm 7.09$ & $87.20 \pm 5.45$ & $92.30 \pm 5.24$ \\
\hline & rs1868092 & $89.62 \pm 5.37$ & $80.04 \pm 3.25$ & $86.39 \pm 4.12$ \\
\hline & rs4953354 & $83.00 \pm 4.24$ & $93.00 \pm 2.55$ & $84.09 \pm 3.46$ \\
\hline \multirow{3}{*}{$\begin{array}{l}\text { Native } \\
\text { Tibetans }\end{array}$} & rs13419896 & $95.00 \pm 1.23$ & $94.15 \pm 2.64$ & $94.65 \pm 2.30$ \\
\hline & rs1868092 & $94.33 \pm 2.08$ & $94.00 \pm 2.04$ & $94.97 \pm 2.54$ \\
\hline & rs4953354 & $96.00 \pm 1.73$ & $94.07 \pm 2.46$ & $94.60 \pm 2.34$ \\
\hline
\end{tabular}

3

4 